\title{
The Correlation Between Symptoms Severity of Left Ventricular Systolic Heart Failure and Tissue Doppler Indices
}

\section{Abstract}

Background: There is no single echo parameter correlates with LVSHF symptoms; however, TDI is a promising tool for assessing both systolic and diastolic heart failure.

Methods: 50 patients with symptomatic LVSHF (EF $\leq 40 \%$ by echocardiography) patients assessed for Functional severity of heart failure according to NYHA classification, Specific activity scale (SAS) test of Goldman, Six minutes walking test, Conventional echocardiographic examination, and TDI for the mitral annulus. The correlation between variables was done using correlation coefficient ( $\mathrm{r}$ ) between heart failure symptoms severity as assessed by NYHA function class (NYHAFC), specific activity scale function Class (SASFC), 6 minutes walking test (6MWT) and echo-tissue Doppler parameters.

Results: There was no significant correlation between 6 minutes walking test and both echo or TDI parameters. There was a significant negative correlation between systolic mitral annular velocity (Sm wave) and both NYHA function class and specific activity scale function class. However, there was no significant correlation with six minutes walking test, and when the study subjects were divided into ICM group and DCM group this correlations persist in the DCM group and lost in the ICM group. There was no significant correlation between systolic heart failure symptoms severity and both Early and late diastolic mitral annular velocities.

Conclusion: Systolic mitral annular motion by TDI (Sm wave) are useful noninvasive method for evaluation of the systolic LV function and correlates well with both NYHAFC and SASFC however, this correlation is significant for dilated non ischemic cardiomyopathy.

Keywords: EF; ICM; DCM; TDI; 6MWT; NYHA; SAS

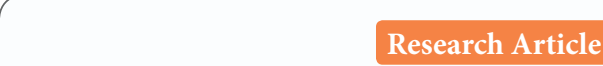

Volume 2 Issue 3 - 2015

Tamer ElKhayat ${ }^{1 *}$, Gamal Aboul Nasr ${ }^{1}$, Sameh Shaheen ${ }^{2}$ and Ayman Mortada ${ }^{2}$

${ }^{1}$ Cardiology Department, National Heart Institute, Egypt ${ }^{2}$ Cardiology Department, Ain Shams University, Egypt

*Corresponding author: Tamer ElKhayat, Cardiology Department, National Heart Institute, 69 Elgoumhoria Street, Port said, Egypt, Tel: +201116850128;

+966549256462; Email: Dr.tamer.el.khayat@gmail.com

Received: February 26, 2014 | Published: March 31, 2015
Abbreviations: 6MWT: Six Minutes Walking Test; A'm: Late Mitral Annular Diastolic Velocity; CMRI: Cardiovascular Magnetic Resonance Imaging; DCM: Dilated Cardiomyopathy; EF: Ejection Fraction; E'm: Early Diastolic Mitral Annular Velocity; HCM: Hypertrophic Cardiomyopathy; ICM: Ischemic Cardiomyopathy; LV: Left Ventricle; NYHA: New York Heart Association; RCM: Restrictive Cardiomyopathy; SAS: Specific Activity Scale; SASFC: Specific Activity Scale Function Class; TDI: Tissue Doppler Imaging

\section{Introduction}

$\mathrm{HF}$ is a complex clinical syndrome that results from any structural or functional impairment of ventricular filling or ejection of blood. The cardinal manifestations of HF are dyspnea and fatigue, which may limit exercise tolerance, and fluid retention, which may lead to pulmonary and/or splanchnic congestion and/or peripheral edema. Data on the incidence of heart failure have consistently demonstrates that heart failure is a common problem, and one that increases markedly with advancing age. The prevalence of heart failure is also increasing as the population ages and the proportion of the population over the age of 65 increases. Ischemic heart disease is currently the most common etiology of chronic heart failure (CHF) in the United States. Primary valvular disease accounts for $7 \%$ of cardiac failure cases, and the majority involves disease of the left-sided cardiac valves. Primary disease of the cardiac muscle can present in a number of forms, three basic forms of functional impairment that have been described (DCM), (HCM) \& (RCM) [1].

There are two types of heart failure patient's exercise performance methods: subjective and objective. The subjective methods include NYHA classification, various activity scales, and quality of life specific questionnaires. The objective methods include various modes of exercise testing to document submaximal exercise capacity (6-minute corridor walk test and anaerobic threshold analysis), as well as maximal exercise capacity (exercise time and maximal oxygen consumption measurement) [2]. A variety of noninvasive imaging modalities are available for assessment of patients with heart failure These include echocardiography, TDI, radionuclide imaging, and cardiovascular magnetic resonance imaging (CMRI), without any correlation to symptoms severity of left ventricular systolic 
heart failure. Therefore, in this study we correlated the clinical severity of symptoms of left ventricular systolic heart failure with Echo-Tissue Doppler Indices of the left ventricular function.

\section{Materials and Methods}

\section{The study subjects}

The subjects of this study were 50 patients with symptomatic left ventricular systolic dysfunction (EF $\leq 40.0 \%$ by echocardiography) attending the heart failure outpatient clinic at the National Heart Institute. The following patients criteria were excluded, atrial fibrillation, End stage Valvular heart disease, unstable angina pectoris, acute heart failure, recent myocardial infarction within 1 month, and technically inadequate echocardiographic images. For each patient the following had been done, Functional severity of heart failure according to NYHA classification, Specific activity scale test of Goldman , Six minutes walking test,

\section{Conventional}

echocardiographic

examination:

Echocardiography was performed with the patients in the left lateral decubitus position. Measurements had been performed according to the recommendations of the American Society of Echocardiography, using Philips EnVisor CHD equipped with 2.5-3 MHZ adult probe.

M-mode tracing: It was obtained to determined (LV) internal dimensions and the percentage of fractional shortening (FS \%) as well as LVEF. Two-dimensional imaging examination was performed in the standard fashion in parasternal long- and short-axis views and apical 4- and 2-chamber views, and TDI for the mitral annulus.

DTI Examination: DTI of the mitral annulus was obtained from the apical (2- and 4-chamber). The sample volume was placed sequentially at the septal and lateral mitral annuli and the resulting velocities were recorded, taking the average of three consecutive beats during normal respiration as follows: peak systolic velocity $(\mathrm{Sm})$, early $(\mathrm{Em})$ and late $(\mathrm{Am})$ diastolic velocities.

\section{Statistics}

Statistical analysis: It was done using SPSS version 17 data expressed as mean \pm standard deviation correlation between variables was done using the Pearson correlation and spearman's rho for nonparametric correlations, significance was considered at $\mathrm{P}$ value $<0.05$. The correlation between variables was done using correlation coefficient (r); a positive $r$ value denotes $+V$ e correlation while a negative $r$ value denotes negative correlation respectively. A correlation (+ve or -Ve) was considered significant at a $\mathrm{P}$ value of $<0.05$.

\section{Results and Discussion}

There was a highly significant positive correlation between NYHA function class and SASFC $(\mathrm{r}=0.726)[\mathrm{P}<0.001]$ and there was a highly significant negative correlation between 6MWT and both NYHA function class $(\mathrm{r}=-0.501)[\mathrm{P}<0.001]$ and SASFC $(\mathrm{r}=$ -0.411) [P=0.003]. Table 1 demonstrates Patient's demographic data and characteristics, and Table 2 shows Echo and TDI patients' characteristics.
Table 1: Patient's demographic data and characteristics.

\begin{tabular}{|c|c|}
\hline Gender & \\
\hline Males & $44(88 \%)$ \\
\hline Females & $6(12 \%)$ \\
\hline Diabetics & $15(30 \%)$ \\
\hline Hypertensive & $12(24 \%)$ \\
\hline Smoker & $23(46 \%)$ \\
\hline IHD & $26(52 \%)$ \\
\hline Dyslipedemic & $18(32 \%)$ \\
\hline Previous PCI & $8(16 \%)$ \\
\hline Previous $\mathrm{CABG}$ & $5(10 \%)$ \\
\hline Characteristics & Number $(\%)$ \\
\hline \multicolumn{2}{|l|}{ NYHA FC } \\
\hline NYHA II & $20(40 \%)$ \\
\hline NYHA III & $28(56 \%)$ \\
\hline NYHA IV & $2(4 \%)$ \\
\hline \multicolumn{2}{|l|}{ SAS FC } \\
\hline SAS II & $21(42 \%)$ \\
\hline SAS III & $17(34 \%)$ \\
\hline SAS IV & $12(24 \%)$ \\
\hline 6MWT & $336 \pm 96 \mathrm{M}$ \\
\hline
\end{tabular}

Table 2: Echo and TDI patients' characteristics.

\begin{tabular}{|c|c|}
\hline Echo parameters & \\
\hline EF & $29 \pm 6 \%$ \\
\hline LVEDD & $7.3 \pm 0.9 \mathrm{~cm}$ \\
\hline LVESD & $6.3 \pm 0.9 \mathrm{~cm}$ \\
\hline TDI parameters & \\
\hline Sm & $5.1 \pm 1.5 \mathrm{~cm} / \mathrm{s}$ \\
\hline E'm & $6.9 \pm 2.6 \mathrm{~cm} / \mathrm{s}$ \\
\hline A'm & $5.7 \pm 2.6 \mathrm{~cm} / \mathrm{s}$ \\
\hline
\end{tabular}

\section{NYHA classification correlations}

There was no significant correlation between NYHA classification and 2D-echo parameters as ejection fraction $(\mathrm{r}=-0.186)$, LVEDD $(\mathrm{r}=-0.002)$, LVESD $(\mathrm{r}=0.047)$. As regard TDI There was a significant negative correlation between NYHA classification and Systolic annular motion ( $\mathrm{S}$ wave) $(\mathrm{r}=-$ 0.312) $[\mathrm{P}=0.027]$ as shown in Figure 1 , however, there was no significant correlation between NYHA classification and early diastolic velocity ( $E^{\prime}$ wave) $(r=-0.260)$ as well as late diastolic velocity ( $A^{\prime}$ wave) $(r=-0.094)$. 


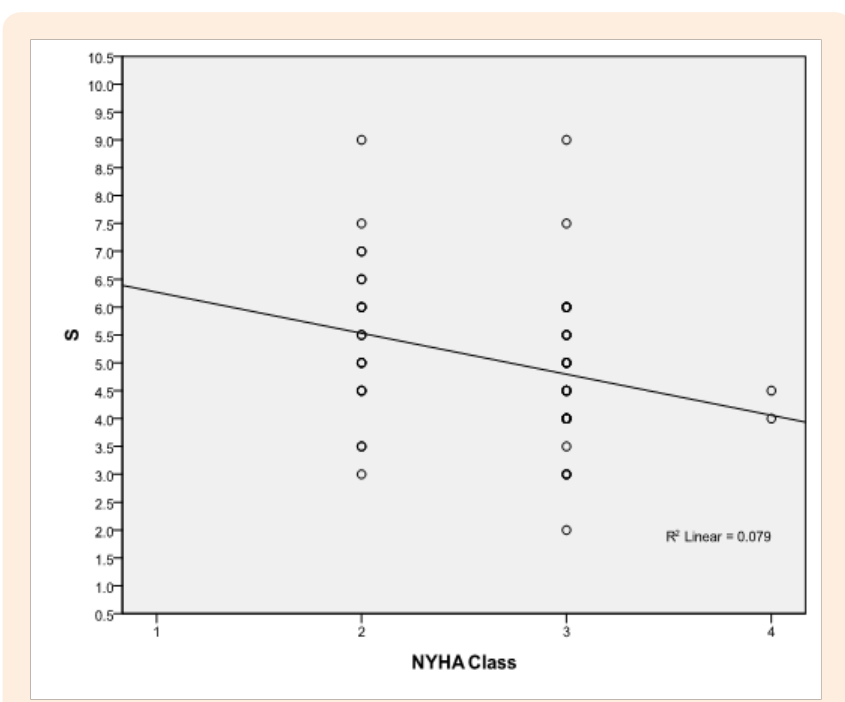

Figure 1: There is a significant negative correlation between NYHA classification and Sm wave in TDI [p=0.027].

\section{Specific activity scale function class (SASFC) correlations}

There was no significant correlation between SASFC and 2D-echo parameters as ejection fraction ( $\mathrm{r}=-0.102)$, LVEDD ( $\mathrm{r}=$ 0.124), LVESD ( $r=-0.093)$, As regard TDI there was a significant negative correlation between SASFC and $\mathrm{S}$ wave velocity ( $\mathrm{r}=-$ 0.301) $[p=0.033]$ as shown in Figure 2, however, there was no significant correlation between SASFC and E' wave velocity $(\mathrm{r}=$ $0.144)$, as well as $A^{\prime}$ wave velocity $(r=-0.080)$.

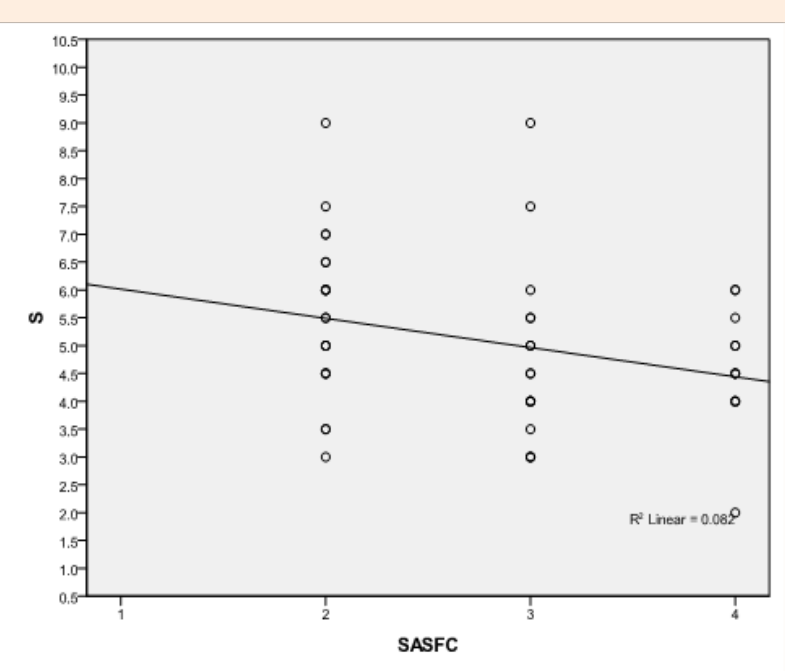

Figure 2: There is a significant negative correlation between Specific Activity Scale function Class and Sm wave in TDI [P=0.033].

Six minutes walking test correlations: There was no significant correlation between Six minutes walking test and 2D-echo parameters as EF ( $\mathrm{r}=0.162)$, LVEDD $(\mathrm{r}=0.008)$, LVESD $(\mathrm{r}=0.001)$. As regard TDI there was no significant correlation between six minutes walking test and TDI parameters as $\mathrm{S}$ wave velocity $(r=0.053), E^{\prime}$ wave velocity $(r=0.191)$, as well as A' wave velocity $(r=-0.216)$. To test whether ischemic etiology would affect the results correlation, based on history of IHD, previous PCI, previous CABG and/or ECG evidence of ischemia patients were subdivided into ICM and DCM groups, 26 patients were ischemic cardiomyopathy while 24 patients were dilated non ischemic cardiomyopathy.

ICM group: In the ICM group patients were all men. Their age ranged from 26 years to 62 years with a mean of $49 \pm 10$ years. There was 12 patients (46.2\%) diabetics, 9 patients (34.6\%) hypertensive, 21 patients $(80.8 \%)$ smokers, and 18 patients $(69.2 \%)$ dyslipedemic. 8 patients $(30.8 \%)$ had previous PCI and 5 patients $(19.2 \%)$ had previous CABG.

NYHA classification correlations: There was no significant correlation between NYHA classification and 2D- echo parameters as EF ( $\mathrm{r}=-0.213)$, LVEDD ( $\mathrm{r}=0.151)$, as well as LVESD $(r=0.125)$. As regard TDI there was no significant correlation between NYHA classification and TDI parameters as $\mathrm{S}$ wave velocity $(\mathrm{r}=-0.141), \mathrm{E}^{\prime}$ wave velocity $(\mathrm{r}=-0.198)$, as well as $\mathrm{A}^{\prime}$ wave velocity $(\mathrm{r}=-0.094)$.

Specific activity scale function class (SASFC) correlations: There was no significant correlation between SASFC and 2D echo parameters as EF ( $\mathrm{r}=-0.019)$, LVEDD ( $\mathrm{r}=0.117)$, LVESD $(\mathrm{r}=0.015)$. As regard TDI there was no significant correlation between SASFC and TDI parameters as $\mathrm{S}$ wave velocity ( $\mathrm{r}=$ $0.221)$, E' wave velocity ( $\mathrm{r}=-0.102)$, as well as $\mathrm{A}^{\prime}$ wave velocity $(\mathrm{r}=0.059)$.

Six minutes walking test correlations: There was no significant correlation between Six minutes walking test and 2D-echo parameters as ejection fraction ( $\mathrm{r}=0.129)$, LVEDD $(r=-0.047)$, LVESD ( $r=-0.047)$. As regard TDI there was no significant correlation between six minutes walking test and TDI parameters as $\mathrm{S}$ wave velocity $(\mathrm{r}=-0.097), \mathrm{E}^{\prime}$ wave velocity $(\mathrm{r}=$ $0.018)$, as well as $A^{\prime}$ wave velocity $(r=-0.255)$.

DCM group: In the DCM group there were 6 females and 18 males, with their age ranged from 22 years to 55 years with a mean of $39 \pm 11$ years, there was 3 patients $(12.5 \%)$ diabetics, 3 patients $(12.5 \%)$ hypertensive, 2 patients $(8.3 \%)$ smokers, their EF ranged from $17 \%$ to $38 \%$ with a mean of $28 \pm 6 \%$.

NYHA classification correlations: There was no significant correlation between NYHA classification and 2D-echo parameters as EF ( $\mathrm{r}=-0.216)$, LVEDD ( $\mathrm{r}=-0.127)$, as well as LVESD $(\mathrm{r}=-0.031)$. As regard TDI There was a highly significant negative correlation between NYHA classification and $S$ wave velocity $(\mathrm{r}=-0.514)[\mathrm{P}=0.010]$ as shown in Figure 3, however, there was no significant correlation between NYHA classification and $\mathrm{E}^{\prime}$ wave velocity $(\mathrm{r}=-0.345)$ as well as $\mathrm{A}^{\prime}$ wave velocity $(\mathrm{r}=-0.165)$.

Specific activity scale function class (SASFC) correlations: There was no significant correlation between SASFC and 2D echo parameters as EF ( $\mathrm{r}=-0.313)$, LVEDD ( $\mathrm{r}=0.385)$, LVESD $(\mathrm{r}=0.213)$. However, there was a significant negative correlation between SASFC and $\mathrm{S}$ wave velocity $(\mathrm{r}=-0.513)[\mathrm{P}=0.010]$ as shown in Figure 4, however, there was no significant correlation between SASFC and E' wave velocity ( $\mathrm{r}=-0.269)$, as well as $\mathrm{A}^{\prime}$ wave velocity $(r=-0.333)$. 


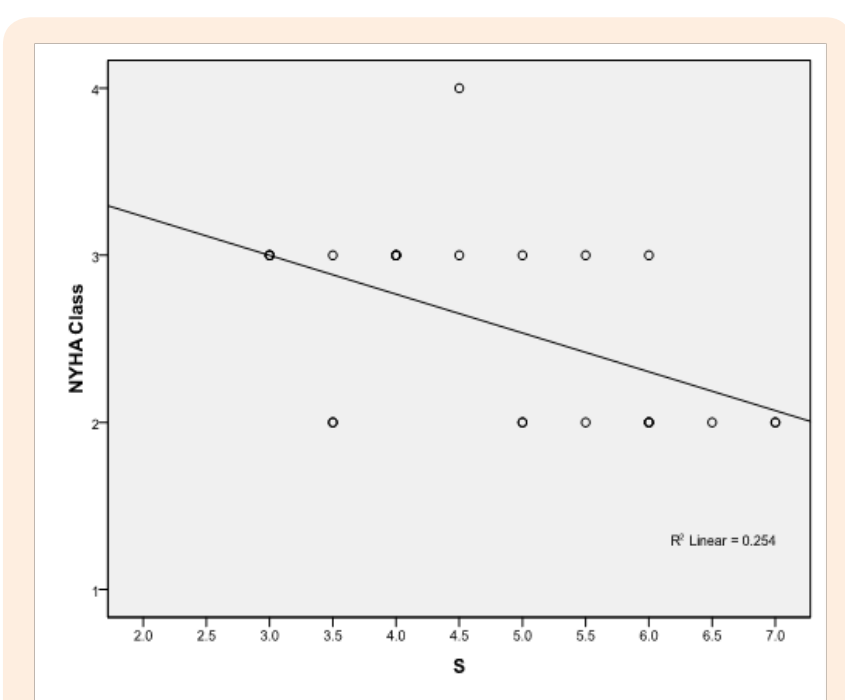

Figure 3: There is strong negative correlation between NYHA classification and Sm wave velocity in DCM group $[\mathrm{P}=0.010]$.

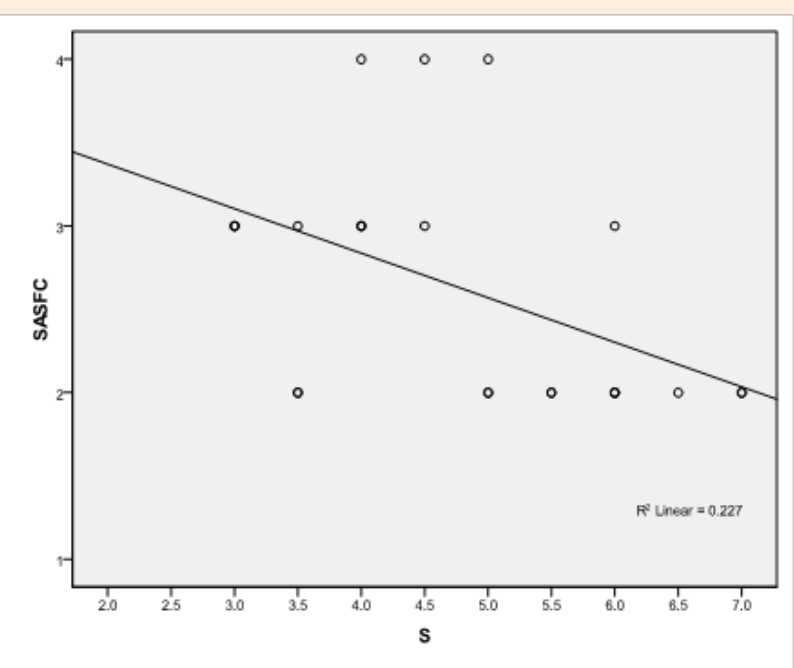

Figure 4: There is a significant negative correlation between specific activity scale and Sm wave velocity in DCM group [P=0.010].

Six minutes walking test correlations: There was no significant correlation between Six minutes walking test and 2D-echo parameters as EF ( $\mathrm{r}=0.213$ ), LVEDD ( $\mathrm{r}=0.094)$, LVESD $(\mathrm{r}=0.077)$. As regard TDI there was no significant correlation between six minutes walking test and TDI parameters as $\mathrm{S}$ wave velocity ( $\mathrm{r}=0.323)$, E' wave velocity $(\mathrm{r}=0.391)$, as well as $\mathrm{A}^{\prime}$ wave velocity ( $\mathrm{r}=-0.158)$.

\section{Discussion}

$\mathrm{LV}$ ejection fraction has been recognized as the most frequent and friendly index to assess cardiac function in both clinical and experimental studies, being relatively easy to measure and to understand, the LV ejection fraction has maintained its position as the most commonly used index. However, it is load dependent and accurate calculation requires measurement of LV enddiastolic and end-systolic volumes; by either echocardiography or LV angiography .

In our study there was no significant correlation between heart failure symptoms severity as assessed by NYHA function class, specific activity scale, 6 minutes walking test and echo parameters of LV ejection e.g. LVEF, LVEDD, LVESD even though when the study subjects were subdivided into ICM group and DCM group still there was no significant correlation between NYHA classification and echo parameters in both groups. The results of this study were in agreement with Cohen et al. [3] \& Smart et al. [4] which showed that EF and LV dimensions did not correlate with HF symptoms, exercise capacity, or myocardial oxygen consumption.

In our study there was a highly significant positive correlation between NYHA function class and SASFC $[\mathrm{P}<0.001]$ and there was a highly significant negative correlation between 6MWT and both NYHA function class $[\mathrm{P}<0.001]$ and SASFC $[\mathrm{P}=0.003]$ and this results was concordant with [5] who showed a similar results correlating Six minute walk test distance with both NYHA and SASFC in 41 heart failure clinic patients, which reflects similarity of the different methods used for the functional assessment of symptoms severity in patients with systolic HF and systolic LV dysfunction.

In our study the Specific Activity Scale (SAS) questions were modified to suite the Egyptian environment e.g. elimination of some questions regarding activities like skiing and playing golf. And when translated from English to Arabic SAS added more power to communication between physician and heart failure patients. Cokkinos et al. [6] used this questionnaire in 74 patients with heart failure in order to assess validity in patients after cardiac transplantation. The questionnaires were found to be valid and reproducible when translated to French. Their findings and ours should encourage more widespread use of these questionnaires as a more valid method for evaluation of the functional state of heart failure patients.

To our knowledge there are no previous studies correlated specific activity scale to echo parameters, however, many studies significantly correlated specific activity scale to other forms of quality of life questionnaire for example living with Heart Failure Questionnaire (MLHF). In our study six minutes walking test correlated poorly with both echo and TDI parameters and this confirmed by a study by Alahdab et al. [7] on 200 patients with heart failure and found that there was no correlation between 6MWT distance walked and LVEF .

Several studies have established a good correlation between the 6MWD and the New York Heart Association (NYHA) functional class, LVEF and health-related quality of life score measured by the Minnesota Living with Heart Failure (MLHF) questionnaire to evaluate the severity of CHF. Our study demonstrated that there was a significant negative correlation between systolic mitral annular velocity (Sm wave) and both NYHA function class $[\mathrm{P}=0.027]$ and specific activity scale function class $[\mathrm{p}=0.033]$ however, there was no significant correlation with six minutes walking test, and when the study subjects were subsequently divided into ICM group and DCM group this correlations persist in the DCM group $[\mathrm{P}=0.010]$ and lost in the ICM group. Suggesting an effect of segmental LV dyskinesia on values of Sm wave velocity measured by TDI for the movement of the mitral 
annulus, and to our knowledge there were no previous studies demonstrating this correlation.

On the other hand Vinereanu et al. [8] showed that in patients with heart failure, the longitudinal peak systolic velocity correlated better with BNP levels than other standard echocardiographic measurements including LVEF and mitral inflow patterns. In our study there was no significant correlation between systolic heart failure symptoms severity and both Early and late diastolic mitral annular velocities denoting non association between early and late LV filling and symptoms severity in patients with HF and systolic LV dysfunction. However, in earlier studies Rihal et al. [9] and Troughton et al. [10] showed significant correlation between LV filling dynamics of the LV and cardiac mortality on follow-up of patients with systolic HF.

On the other hand, Wang et al. [11] found that the early diastolic mitral annular velocity was reduced from an early stage of reduced LV systolic function and remained reduced even with increased filling pressure which has an incremental prognostic power over what LVEF can provide, with a worse prognosis on long term follow-up [12-16].

\section{Conclusion}

In spite of their difference in methodology for evaluation of severity of the degree of HF in patients with LV systolic dysfunction (from totally subjective e.g. NYHA \& SAS to totally objective e.g. 6MWT), all are strongly correlated with each other and can be used interchangeably as accurate assessment of severity of HF [17-20]. The Arabic translation of the Specific Activity Scale was shown to be very helpful in both proper evaluation of symptoms severity of HF and improving patient physician relationship in this group of patients. Systolic mitral annular motion by TDI (Sm wave) are useful non invasive method for evaluation of the systolic LV function and correlates well with both NYHA function class and specific activity scale function class however, this correlation is significant for dilated non ischemic cardiomyopathy [21,22].

\section{Study Limitations}

Our study has few limitations, first, the study population was relatively small and additional future studies with larger patients' population would be needed. Second, in subdividing systolic heart failure patients into ICM and DCM, although, we were dependant on history and non invasive tests of myocardial ischemia, no routine coronary angiography was done. Finally, in spite of finding no correlation between HF subjects' evaluation and LVEF, this parameter was shown in previous studies to have prognostic correlation in patients with HF. We suggest a future study involving larger number of patients with long term follow up to test this hypothesis.

\section{References}

1. Clyde W Y, Mariell J, Biykem B, Javed B, Donald E C, et al. (2013) 2013 ACCF/AHA Heart Failure Guideline. J Am Coll Cardiol 62(16): e147-e239.

2. Mariell L, Evan L (2003) Heart Failure a Clinician's Guide to Ambulatory Diagnosis and Treatment. Humana Press 1: 163-208.

3. Cohen-Solaol A, Tabet JY, Logeart D, Bourgoin P, Tokmakova M, et al. (2002) A non-invasively determined surrogate of cardiac power ('circulatory power') at peak exercise is a powerful prognostic factor in chronic heart failure. Eur Heart J 23(10): 806-814.

4. Smart N, Haluska B, Leano R, Case C, Mottram PM, et al. (2005) Determinants of functional capacity in patients with chronic heart failure: role of filling pressure and systolic and diastolic function. Am Heart J 149(1): 152-158.

5. Basuray, Gordon, Ferrara, Mezgarzedeh, Mohamedali B, et al. (2008) Patient Reported Symptom Activity Scale and Correlation to the Six Minute Walk Test in an Outpatient Heart Failure Population. Journal of Cardiac Failure 14(6): S107.

6. Cokkinos DV (1999) The value of questionnaires in assessing physical activity, fitness and quality of life. Heart Failure Reviews 3: 305-311.

7. Alahdab M, Ibrahaim N, Sirikarn N (2009) Six Minute Walk Test Predicts Long-Term All-Cause Mortality and Heart Failure Rehospitalization in African-American Patients Hospitalized With Acute Decompensated Heart Failure. J Card Fail 15(2): 130-135.

8. Vinereanu D, Lim PO, Frenneaux MP (2005) Reduced myocardial velocities of left ventricular long-axis contraction identify both systolic and diastolic heart failure-a comparison with brain natriuretic peptide. Eur J Heart Fail 7(2): 512-519.

9. Rihal CS, Nishimura RA, Hatle LK, Bailey KR, Tajik AJ (1994) Systolic and diastolic dysfunction in patients with clinical diagnosis of dilated cardiomyopathy. Relation to symptoms and prognosis. Circulation 90(6): 2772-2779.

10. Troughton RW, Prior DL, Pereira JJ, Martin M, Fogarty A, et al. (2004) Plasma B-type natriuretic peptide levels in systolic heart failure: importance of left ventricular diastolic function and right ventricular systolic function. J Am Coll Cardiol 43(3): 416-422.

11. Wang M, Yip G, Yu CM, Zhang Q Zhang Y, et al. (2005) Independent and incremental prognostic value of early mitral annulus velocity in patients with impaired left ventricular systolic function. J Am Coll Cardiol 45(2): 272-277.

12. Levy D, Kenchaiah S, Larson MG, Benjamin EJ, Kupka MJ, et al. (2002) Long-term trends in the incidence of and survival with heart failure. N Engl J Med 347(18): 1397-1402.

13. Butler RN (1997) Population aging and health. BMJ 315(7115): 1082-1084.

14. Ishihara K, Zile MR, Kanazawa S, Tsutsui H, Urabe Y, et al. (1992) Left ventricular mechanics and myocyte function after correction of experimental chronic mitral regurgitation by combined mitral valve replacement and preservation of the native mitral valve apparatus. Circulation 86(5 Suppl): II16-II25.

15. Peter L, Robert O, Douglas L, Douglas P, Eugene B, et al. (2007) Braunwald's Heart Disease: A Textbook of Cardiovascular Medicine. (8 ${ }^{\text {th }}$ Edn), pp. 561-582.

16. Goldman L, Hashimoto B, Cook EF, Loscalzo A (1981) Comparative reproducibility and validity of systems for assessing cardiovascular functional class: advantages of a new specific activity scale. Circulation 64(6): 1227-1234

17. Schiller NB, Shah PM, Crawford M, DeMaria A, Devereux R, et al. (1989) Recommendations for quantitation of the left ventricle by 2-dimensional echocardiography. J Am Soc Echo-cardiogr 2(5): 358-367.

18. John E Sanderson, Alan G Fraser (2006) Systolic Dysfunction in Heart Failure with a Normal Ejection Fraction: Echo-Doppler Measurements. Prog Cardiovasc Dis 49(3): 196-206.

19. Chryssanthopoulos SN, Dritsas A, Cokkinos DV (2005) Activity 
questionnaires; a useful tool in accessing heart failure patients. Int J Cardiol 105(3): 294-299.

20. Bittner V, Weiner DH, Yusuf S, Rogers WJ, McIntyre KM, et al. (1993) Prediction of mortality and morbidity with a 6-minute walk test in patients with left ventricular dysfunction. JAMA 270(14): 17021707.

21. Miyamoto S, Nagaya N, Satoh T, Kyotani S, Sakamaki F, et al. (2000)
Clinical correlates and pronostic significance of six-minute walk test in patients with pulmonary hypertension. Am J Respir Crit Care Med 161(2 Pt 1): 487-492.

22. Shah M, Hasselblad V, Gheorghiade M, Adams KF, Swedberg K, et al. (2001) Prognostic usefulness of the six-minute walk in patients with advanced congestive heart failure secondary to ischemic or nonischemic cardiomyopathy. Am J Cardiol 88(9): 987-993. 